\title{
Cerebral Perfusion and Gray Matter Changes Associated With Chemotherapy-Induced Peripheral Neuropathy
}

Kelly N.H. Nudelman, Brenna C. McDonald, Yang Wang, Dori J. Smith, John D. West, Darren P. O'Neill, Noah R. Zanville, Victoria L. Champion, Bryan P. Schneider, and Andrew J. Saykin

See accompanying editorial on page 649

Kelly N.H. Nudelman, Brenna C. McDonald, Yang Wang, Dori J. Smith, John D. West, Darren P. O'Neill, Victoria L. Champion, Bryan P. Schneider, and Andrew J. Saykin, Indiana University School of Medicine; Noah R. Zanville and Victoria L. Champion, Indiana University School of Nursing, Indianapolis, IN; and Yang Wang, Medical College of Wisconsin, Milwaukee, WI.

Published online ahead of print at www.jco.org on November 2, 2015

Supported, in part, by the National Cancer Institute (Grants No. R01 CA101318, R01 CA082709, and R25 CA117865), the National Institute on Aging (Grants No. R01 AG19771 and P30 AG10133), and the Eunice Kennedy Shriver National Institute of Child Health and Human Development (Grant No. U54 HD062484) of the National Institutes of Health; the Indiana Clinical and Translational Sciences Institute (Grants No. UL1 RR025761, RR027710 01, and RR020128); and an Indiana University Melvin and Bren Simon Cancer Center American Cancer Society institutional grant.

The content of this article is solely the responsibility of the authors and does not necessarily represent the official views of the National Institutes of Health.

Authors' disclosures of potential conflicts of interest are found in the article online at www.jco.org. Author contributions are found at the end of this article.

Corresponding author: Andrew J. Saykin, PsyD, Center for Neuroimaging, Department of Radiology and Imaging Sciences, Indiana University School of Medicine, 355 W 16th St, GH Ste 4100 Indianapolis, IN 46202; e-mail: asaykin@iupui.edu.

(C) 2015 by American Society of Clinical Oncology

0732-183X/16/3407w-677w/\$20.00

DOI: $10.1200 / J C O .2015 .62 .1276$

\section{$\begin{array}{llllllll}\text { A } & \text { B } & \text { S } & \text { T } & \text { R } & \text { A } & \text { C } & \text { T }\end{array}$}

\section{Purpose}

To investigate the longitudinal relationship between chemotherapy-induced peripheral neuropathy (CIPN) symptoms (sx) and brain perfusion changes in patients with breast cancer. Interaction of CIPN-sx perfusion effects with known chemotherapy-associated gray matter density decrease was also assessed to elucidate the relationship between CIPN and previously reported cancer treatment-related brain structural changes.

\section{Methods}

Patients with breast cancer treated with $(n=24)$ or without ( $n=23$ ) chemotherapy underwent clinical examination and brain magnetic resonance imaging at the following three time points: before treatment (baseline), 1 month after treatment completion, and 1 year after the 1-month assessment. CIPN-sx were evaluated with the self-reported Functional Assessment of Cancer Therapy/ Gynecologic Oncology Group-Neurotoxicity four-item sensory-specific scale. Perfusion and gray matter density were assessed using voxel-based pulsed arterial spin labeling and morphometric analyses and tested for association with CIPN-sx in the patients who received chemotherapy.

\section{Results}

Patients who received chemotherapy reported significantly increased CIPN-sx from baseline to 1 month, with partial recovery by 1 year $(P<.001)$. CIPN-sx increase from baseline to 1 month was significantly greater for patients who received chemotherapy compared with those who did not $(P=.001)$. At 1 month, neuroimaging showed that for the group that received chemotherapy, CIPN-sx were positively associated with cerebral perfusion in the right superior frontal gyrus and cingulate gyrus, regions associated with pain processing $(P<.001)$. Longitudinal magnetic resonance imaging analysis in the group receiving chemotherapy indicated that CIPN-sx and associated perfusion changes from baseline to 1 month were also positively correlated with gray matter density change $(P<.005)$.

\section{Conclusion}

Peripheral neuropathy symptoms after systemic chemotherapy for breast cancer are associated with changes in cerebral perfusion and gray matter. The specific mechanisms warrant further investigation given the potential diagnostic and therapeutic implications.

\section{J Clin Oncol 34:677-683. (C) 2015 by American Society of Clinical Oncology}

\section{INTRODUCTION}

Chemotherapy-induced peripheral neuropathy (CIPN) is a common, potentially permanent adverse effect of breast cancer treatment. ${ }^{1,2}$ In a metaanalysis of CIPN across many cancer types in 31 studies, CIPN was observed in $61 \%$ of patients 1 month after treatment, $60 \%$ of patients 3 months after treatment, and $30 \%$ of patients 6 months after treatment, indicating high prevalence as well as persistence of symptoms in a subgroup of patients. ${ }^{3}$ CIPN may necessitate cancer treatment alterations, and currently, there are no effective treatments for symptoms. ${ }^{4}$ Given the prevalence as well as evidence that CIPN may interfere with survivor quality of life, ${ }^{4}$ further characterization of the biologic mechanisms driving CIPN may suggest potential novel approaches to treatment.

Despite various studies reporting changes in brain structure and function associated with other types of pain, ${ }^{5-10}$ the impact of CIPN on brain structure and function has not been well studied. To date, there has been only one publication on this topic, which compared patients with multiple myeloma with CIPN to controls and found significant 
differences in CIPN-associated brain activation during pain processing. ${ }^{11}$ The relationship between CIPN and cerebral perfusion has not been specifically studied to date but could be valuable in identifying future CIPN treatment targets. We hypothesized that CIPN symptoms (CIPN-sx) in patients with breast cancer would be longitudinally associated with altered resting state cerebral perfusion. In addition, in view of our previous observations of altered cerebral perfusion and gray matter density after breast cancer chemotherapy treatment, ${ }^{12-14}$ we investigated how these changes might relate to any CIPN-associated cerebral perfusion change.

\section{METHODS}

\section{Participants}

Written informed consent was obtained from all study participants using a protocol approved by the Indiana University Institutional Review Board in accordance with the Declaration of Helsinki. The female breast cancer cohort used for this study has been extensively described in previous publications. ${ }^{13,14}$ For the current analyses, the sample consisted of patients treated with $(\mathrm{n}=24)$ or without $(\mathrm{n}=23)$ common, standard-dose chemotherapy regimens. Baseline assessment data were collected after surgery and before chemotherapy treatment for most individuals. Eight patients received neoadjuvant chemotherapy after their baseline assessment but before any definitive surgical treatment; these individuals did not differ significantly from the other patients who received chemotherapy regarding demographic factors, depression, or anxiety. The same assessments were also collected 1 month $(1 \mathrm{M})$ after chemotherapy treatment completion and 1 year (1Y) later (13 months after treatment), with yoked intervals for patients who did not receive chemotherapy. Group sizes were smaller (chemotherapy, $\mathrm{n}=18$; no chemotherapy, $\mathrm{n}=19$ ) for 1 Y analyses as a result of missing CIPN-sx data and participant exclusion (discussed later). Only patients with noninvasive (stage 0 ) or nonmetastatic invasive (stage I, II, or III) disease were included. Besides metastatic disease, additional exclusion criteria for all participants included prior cancer, substance abuse, and other medical, neurologic, and psychiatric risk factors that might affect cerebral structure or function, as described in McDonald et al. ${ }^{13}$

Table 1. Cohort Demographic and Clinical Characteristics, Rating Scales, and Cancer Treatment Data

\begin{tabular}{|c|c|c|c|}
\hline Characteristic & $\begin{array}{l}\text { Patients Who Received Chemotherapy } \\
\qquad(1 \mathrm{M}, \mathrm{n}=24 ; 1 \mathrm{Y}, \mathrm{n}=18)\end{array}$ & $\begin{array}{l}\text { Patients Who Did Not Receive } \\
\text { Chemotherapy }(1 \mathrm{M}, \mathrm{n}=23 \\
\qquad 1 \mathrm{Y}, \mathrm{n}=19)\end{array}$ & $P$ \\
\hline Mean BL age, years (SD) & $49.4(7.9)$ & $52.0(9.1)$ & $.312^{*}$ \\
\hline Mean BL education, years (SD) & $15.3(2.9)$ & $15.4(2.3)$ & $.985^{*}$ \\
\hline Mean BL estimated full-scale IQ, Barona Index, ${ }^{15}$ (SD) & $109.9(6.9)$ & $111.2(5.7)$ & $.501^{*}$ \\
\hline White non-Hispanic, No. (\%) & 19 (79\%) & $21(91 \%)$ & $.226+$ \\
\hline \multicolumn{4}{|l|}{ CES-D score, mean (SD) } \\
\hline$B L$ & $9.2(7.8)$ & $7.3(7.7)$ & $.392^{*}$ \\
\hline $1 \mathrm{M}$ & $13.9(8.4)$ & $9.4(9.6)$ & $.088^{*}$ \\
\hline $1 Y$ & $10.9(7.5)$ & $8.3(9.9)$ & $.401^{*}$ \\
\hline \multicolumn{4}{|l|}{ STAI-S score, mean (SD) } \\
\hline $\mathrm{BL}$ & $33.4(13.9)$ & $27.8(7.7)$ & $.098^{*}$ \\
\hline $1 \mathrm{M}$ & $34.8(12.0)$ & $31.2(12.7)$ & $.332^{*}$ \\
\hline $1 \mathrm{Y}$ & $30.8(8.2)$ & $28.8(9.4)$ & $.498^{*}$ \\
\hline Mean $\mathrm{BL}$ to $1 \mathrm{M}$ interscan interval, days (SD) & $152.2(63.1)$ & $178.7(74.1)$ & $.192^{*}$ \\
\hline Mean $1 \mathrm{M}$ to $1 \mathrm{Y}$ interscan interval, days (SD) & $379.5(20.7)$ & $371.8(11.8)$ & $.170^{*}$ \\
\hline \multicolumn{4}{|l|}{ Cancer stage, No. of patients (\%) } \\
\hline 0 (DCIS) & $0(0)$ & $5(22)$ & $.002 \dagger$ \\
\hline I & $11(46)$ & $16(70)$ & \\
\hline$\|$ & $9(38)$ & $2(9)$ & \\
\hline III & $4(17)$ & $0(0)$ & \\
\hline \multicolumn{4}{|l|}{ Therapy, No. of patients (\%) } \\
\hline Received radiotherapy by $1 \mathrm{M}$ & $2(8)$ & $15(65)$ & $<.001 \dagger$ \\
\hline Received radiotherapy by $1 \mathrm{Y}$ & $11(61)$ & $12(63)$ & $.020 \dagger$ \\
\hline On antiestrogen therapy at $\mathrm{BL} \neq$ & $0(0)$ & $1(4)$ & $.498 \dagger$ \\
\hline On antiestrogen therapy at $1 \mathrm{M} \neq$ & $3(13)$ & $16(70)$ & $<.001 \dagger$ \\
\hline On antiestrogen therapy at $1 \mathrm{Y} \ddagger$ & $8(44)$ & $15(79)$ & $.033 \dagger$ \\
\hline \multicolumn{4}{|l|}{ Chemotherapy regimen§ } \\
\hline DOX/CYC/paclitaxel & 7 (29) & & \\
\hline $\mathrm{DOC} / \mathrm{CYC}$ & $6(25)$ & & \\
\hline DOC/carboplatin & $6(25)$ & & \\
\hline $\mathrm{DOC} / \mathrm{DOX} / \mathrm{CYC}$ & $2(8)$ & & \\
\hline DOC/cisplatin & $1(4)$ & & \\
\hline DOX/CYC & 1 (4) & & \\
\hline Paclitaxel & $1(4)$ & & \\
\hline \multicolumn{4}{|c|}{$\begin{array}{l}\text { NOTE. Values calculated at BL unless otherwise noted. } \\
\text { Abbreviations: } 1 \mathrm{M}, 1 \text { month after chemotherapy treatment completion; } 1 \mathrm{Y}, 1 \text { year after } 1 \mathrm{M} \text { time point; BL, baseline (prechemotherapy treatment); CES-D, Center for } \\
\text { Epidemiologic Studies-Depression; CYC, cyclophosphamide; DOC, docetaxel; DOX, doxorubicin; STAI-S, State Trait Anxiety Inventory-State. } \\
{ }^{*} \text { Analysis of variance. } \\
+\chi^{2} \text { test. } \\
\text { fAntiestrogen therapies included tamoxifen (majority), anastrozole, letrozole, exemestane, raloxifene, and leuprolide acetate. } \\
\text { } \text { Eight patients were also treated with trastuzumab, one was also treated with sunitinib, and one was also treated with bevacizumab. }\end{array}$} \\
\hline
\end{tabular}




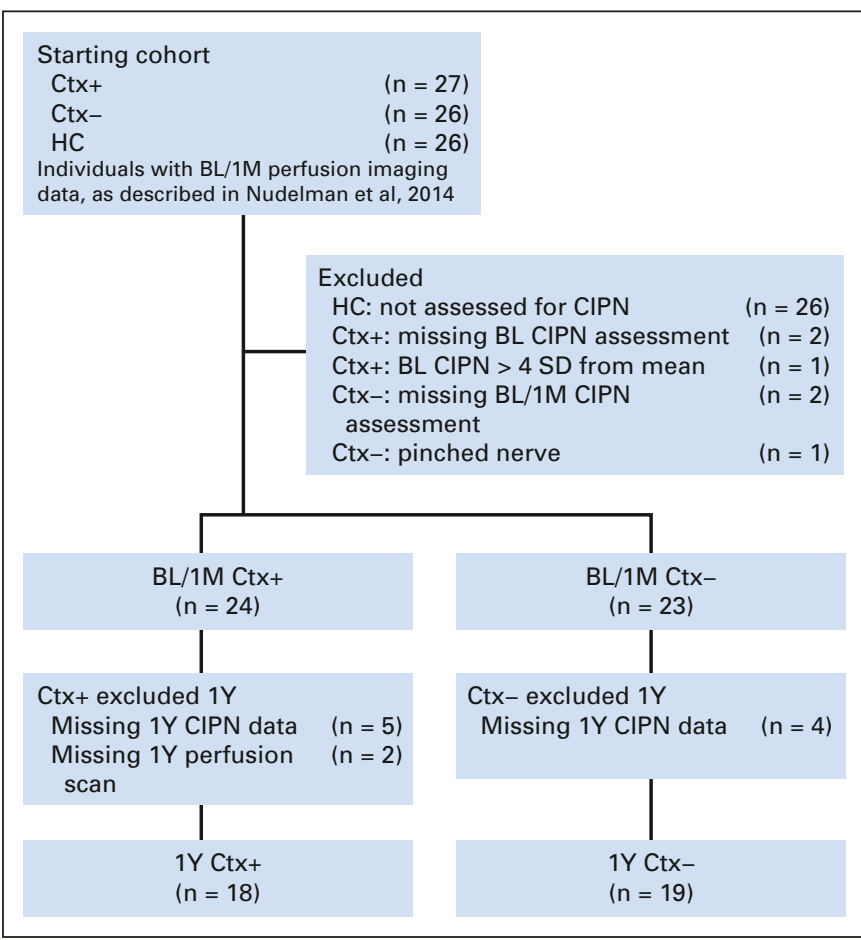

Fig 1. CONSORT diagram. Number of individuals considered at each stage of the study, as well as number and reasons for excluded individuals. $1 \mathrm{M}, 1$ month after chemotherapy treatment completion (or yoked interval for patients who did not receive chemotherapy and controls); $1 \mathrm{Y}, 1$ year after $1 \mathrm{M}$ time point; $\mathrm{BL}$, baseline (before chemotherapy treatment for the majority of patients); CIPN chemotherapy-induced peripheral neuropathy; $\mathrm{Ctx}+$, patients with breast cancer treated with chemotherapy; $\mathrm{CTx}-$, patients with breast cancer treated without chemotherapy; $\mathrm{HC}$, healthy controls; SD, standard deviation.

Demographic and treatment characteristics are listed in Table 1. The Center for Epidemiologic Studies-Depression Scale ${ }^{16}$ and the State Trait Anxiety Inventory-State subscale ${ }^{17}$ were used to measure depressive symptoms and anxiety. Between-group comparisons including analysis of variance (ANOVA), general linear models, and $\chi^{2}$ tests were run with SPSS Statistics 21 (IBM Corporation, Somers, NY).

CIPN-sx were assessed using the self-report, validated Functional Assessment of Cancer Therapy/Gynecologic Oncology Group-Neurotoxicity (FACT/GOG-Ntx) four-item sensory-specific subscale. ${ }^{18}$ Summed scores for each time point were generated. Given the specific nature of each included question, missing data were not imputed; if any question was unanswered, CIPN-sx total score was counted as missing. Exclusions from the cohort presented in Nudelman et $\mathrm{al}^{14}$ comprised the following: for patients who received chemotherapy, two individuals were missing baseline CIPN-sx, and an additional six individuals were missing 1Y CIPN-sx; for patients who did not receive chemotherapy, two individuals were missing baseline or $1 \mathrm{M}$ CIPN-sx, and an additional four individuals were missing 1Y CIPN-sx. Two additional individuals were excluded based on high baseline CIPN-sx: one patient who received adjuvant chemotherapy had CIPN-sx more than 4 standard deviations from the group mean at baseline, whereas one patient who did not receive chemotherapy with the highest CIPN-sx baseline score was noted to have a pinched nerve, requiring pain medication. Exclusion of these individuals yielded the final group sizes, presented earlier as well as in the CONSORT diagram (Fig 1).

Repeated measures ANOVA in SPSS was used to analyze CIPN-sx change over all three time points. In addition, ANOVA analyses at each time point contrasted CIPN-sx means between treatment groups. Finally, $1 \mathrm{M}$ - baseline CIPN-sx scores were analyzed in a two-way ANOVA to test for treatment group differences in change from baseline to after treatment.

\section{Magnetic Resonance Imaging Acquisition}

Cerebral perfusion scans were acquired with a Siemens Tim Trio 3T whole-body magnetic resonance imaging scanner (Siemens, Munich, Germany) using a 12-channel receiver-only head coil. During scan acquisition, patients were in a conscious resting state with closed eyes. Details of the scanning protocol used to obtain cerebral perfusion measurements have been previously published. ${ }^{19}$ Briefly, a Q2TIPS pulsed arterial spin labeling sequence was applied using the proximal inversion with a control for offresonance effects labeling scheme. Labeling was performed with an adiabatic inversion pulse with a $10-\mathrm{cm}$ labeling region and $25-\mathrm{mm}$ spacing from the distal edge of the labeled region to the image section, followed by optimized inversion time delays $\left(\mathrm{TI}_{1}=700\right.$ milliseconds and $\mathrm{TI}_{2}=1,800$ milliseconds). These time delays were chosen to minimize 3-T intravascular signal intensity. Images were acquired using a gradient-echo single shot echo planar imaging readout with the following acquisition parameters: repetition time/echo time $=3,000 / 13$ milliseconds, field of view $=224 \mathrm{~mm}$, and matrix $=64 \times 64$. The imaging region consisted of 16 contiguous ascending axial slices of 7-mm thickness. Each perfusion measurement consists of 100 dynamic images (50 control and label image pairs) plus one $\mathrm{M}_{0}$ image (the equilibrium brain tissue magnetization used to normalize the difference perfusion map), requiring a scan time of about 5 minutes. Head motion artifact was minimized using the scanner's built-in three-dimensional online prospective acquisition correction. High-resolution T1-weighted magnetization prepared rapid gradient echo (MPRAGE) images and high-resolution echo planar imaging whole-brain scans were acquired for subsequent reference and normalization. T2-weighted and fluid-attenuated inversion recovery sequences were acquired to check for incidental pathology. The high-resolution MPRAGE series for the analysis of gray matter density was acquired as previously described. ${ }^{13}$

\section{Image Processing}

Pulsed arterial spin labeling magnetic resonance imaging scan processing was performed using previously described methods. ${ }^{19}$ Briefly, matched control images were subtracted from labeled images to create a perfusionweighted time series; the results were used to create quantitative regional perfusion maps for each scan, which were normalized to Montréal Neurologic Institute space using SPM8 (Wellcome Department of Cognitive Neuroscience, London, United Kingdom; http://www.fil.ion.ucl.ac.uk/spm/software/ $\mathrm{spm} 8 /$ ). Scans were resampled to $2-\mathrm{mm}^{3}$ voxels and smoothed with a $6 \times 6$

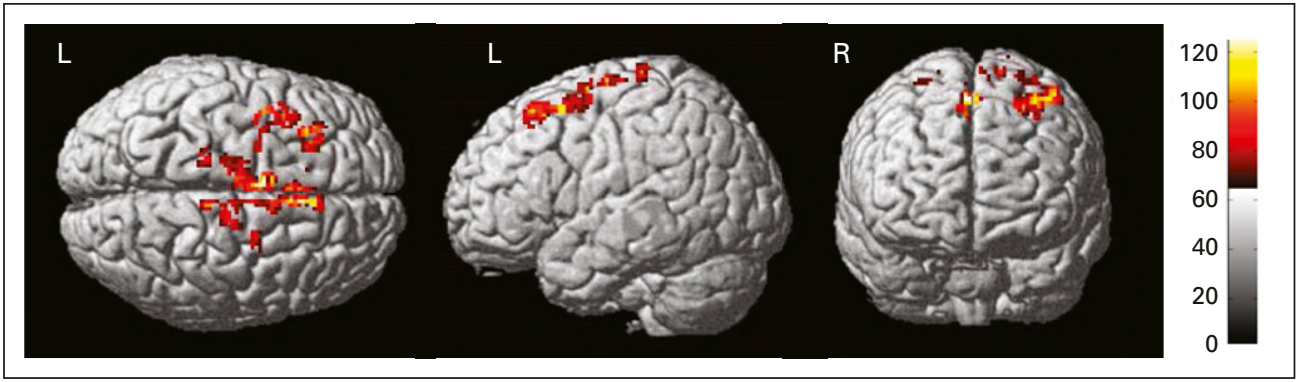

Fig 2. Chemotherapy-induced peripheral neuropathy symptoms (CIPN-sx) positively associated with perfusion. Surface rendering of the positive association of 1-month post-treatment perfusion with level of CIPN-sx reported at 1 month after treatment $\left(P_{\text {crit }}<.001, \mathrm{k}=25\right)$; colored regions indicate increasing statistical significance, as shown on the color scale. 
Table 2. CIPN-sx FACT/GOG-Ntx Four-Item Subscale Group Mean Values

\begin{tabular}{|c|c|c|c|}
\hline \multirow[b]{2}{*}{ Time Period } & \multicolumn{2}{|c|}{ Mean (SD) } & \multirow[b]{2}{*}{$P^{*}$} \\
\hline & $\begin{array}{l}\text { Patients Who Received Chemotherapy } \\
\qquad(1 \mathrm{M}, \mathrm{n}=24 ; 1 \mathrm{Y}, \mathrm{n}=18)\end{array}$ & $\begin{array}{l}\text { Patients Who Did Not Receive Chemotherapy } \\
\qquad(1 \mathrm{M}, \mathrm{n}=23 ; 1 \mathrm{Y}, \mathrm{n}=19)\end{array}$ & \\
\hline BL CIPN-sx & $0.46(0.9)$ & 1.09 (2.8) & .250 \\
\hline 1M CIPN-sx & $4.71(5.0)$ & 0.91 (1.4) & .001 \\
\hline $1 Y \mathrm{CIPN}-\mathrm{sx}$ & $4.06(3.4)$ & $1.79(2.5)$ & .027 \\
\hline$P+$ & $<.001$ & .301 & \\
\hline
\end{tabular}

$\times 8 \mathrm{~mm}$ full width at half maximum kernel. Image processing of the MPRAGE series for analysis of gray matter density was performed as previously described. ${ }^{13}$

\section{Image Analysis}

To examine the potential roles of cerebral perfusion and gray matter in CIPN-related pain processing, all imaging analyses were limited to the group that received chemotherapy. First, time point analysis was performed to test for association of cerebral perfusion at $1 \mathrm{M}$ and $1 \mathrm{Y}$ with CIPN-sx at $1 \mathrm{M}$ and $1 \mathrm{Y}$, respectively. For each time point, whole-brain perfusion scans were tested for association with the CIPN-sx variable using voxel-wise multiple regression imaging analysis in SPM8. A mask of regions of CIPN-sx-related perfusion at $1 \mathrm{M}$ was created for later use.

Second, baseline to $1 \mathrm{M}$ longitudinal analysis was performed to test for association of cerebral perfusion changes with CIPN-sx. Perfusion changes (1M - baseline scan images, obtained using the ImCalc utility in SPM8) were tested for association with CIPN-sx at 1M covarying for baseline, using voxelwise multiple regression imaging analysis in SPM8. CIPN-sx scores at $1 \mathrm{M}$ covaried for baseline were used to examine the relationship between perfusion change and symptom severity, controlling for variance related to higher baseline symptom report unrelated to chemotherapy treatment. Mean values for the cluster identified in this analysis were extracted using MarsBar in SPM8, and $1 \mathrm{M}$ - baseline values were computed in SPSS to obtain CIPN-sxassociated perfusion cluster value changes ${ }^{20}$ for later use.

Third, correlation analysis was performed for CIPN-sx and related perfusion changes with gray matter density frontal changes (reported in $\mathrm{McDonald}$ et $\mathrm{al}^{13}$ ). Mean values for the two frontal gray matter density clusters were obtained for each participant using MarsBar in SPM8. SPSS was then used to create an average of the two clusters for each participant, and $1 \mathrm{M}$ - baseline values were computed to obtain a single value for combined cluster change over time for each individual. Gray matter density cluster value changes were tested using SPSS for Pearson correlation with baseline to $1 \mathrm{M}$ changes in both CIPN-sx scores and perfusion cluster values (obtained in the second imaging analysis).

Fourth, image masking of the previously reported gray matter densityassociated frontal perfusion decrease ${ }^{14}$ observed in this cohort was performed to determine whether it occurred in CIPN-sx-related regions identified in the first imaging analysis. Multiple regression analysis of perfusion changes $(1 \mathrm{M}-$ baseline scans, as in the second imaging analysis) with gray matter density cluster value baseline to $1 \mathrm{M}$ changes in SPM8 was masked with 1M CIPN$\mathrm{sx}$-related perfusion (from the first imaging analysis).

For the first three imaging analyses, to determine statistical significance and reduce noise, the voxel-wise critical significance threshold $\left(P_{\text {crit }}\right)$ was set to .001 uncorrected, with a minimum cluster extent $(\mathrm{k})$ of 25 voxels. For the fourth analysis, the uncorrected voxel-wise critical significance threshold was relaxed to $P_{\text {crit }}<.01, \mathrm{k}=25$, to identify any changes within the masked area. As presented in Nudelman et $\mathrm{al}^{14}{ }^{14}$ demographic and other confounding variables such as caffeine consumption were previously considered for their potential impact on imaging analyses. These variables were not shown to be significant and are not included in the current analyses.

\section{RESULTS}

\section{Demographic Analysis}

Comparison of treatment factors, including radiation, antiestrogen therapy, and chemotherapy, as well as cancer stage, identified significant group differences (Table 1); given current treatment protocols, these differences are typical for this type of study. At baseline, there were no significant differences between groups in terms of age, education, intelligence quotient, and race/ethnicity. In addition, although a trend was observed for increased post-treatment depression in the chemotherapy
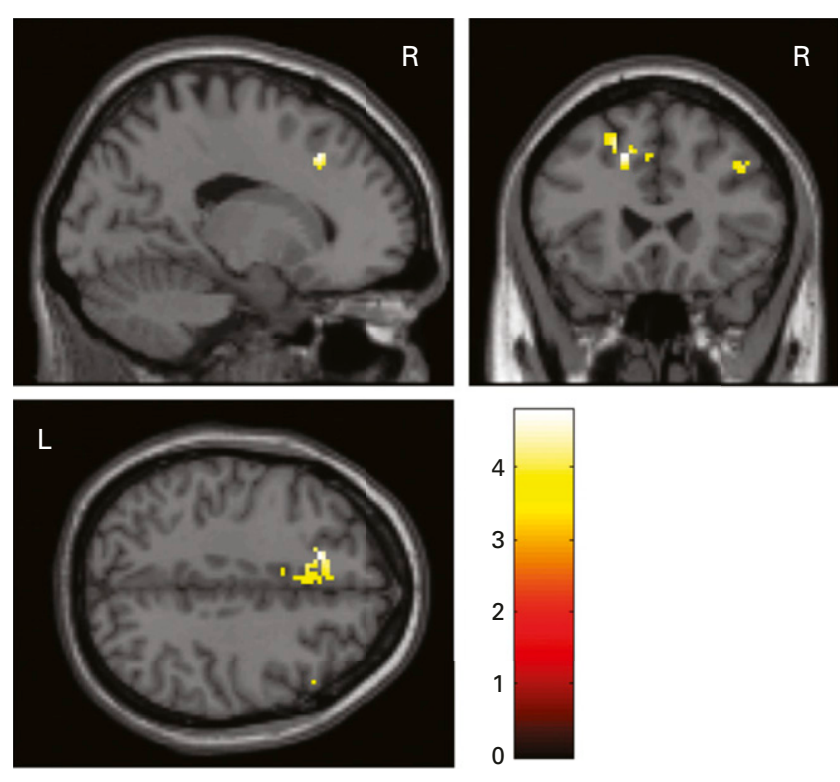

Fig 3. Chemotherapy-induced peripheral neuropathy symptoms (CIPN-sx) associated with perfusion increase. Colored regions of brain sections show areas with statistically significant association of baseline (before chemotherapy treatment) to 1-month post-treatment perfusion increase and 1-month post-treatment CIPN-sx covarying for baseline $\left(P_{\text {crit }}<.001, \mathrm{k}=25\right)$; colored regions indicate increasing statistical significance, as shown on the color scale. 

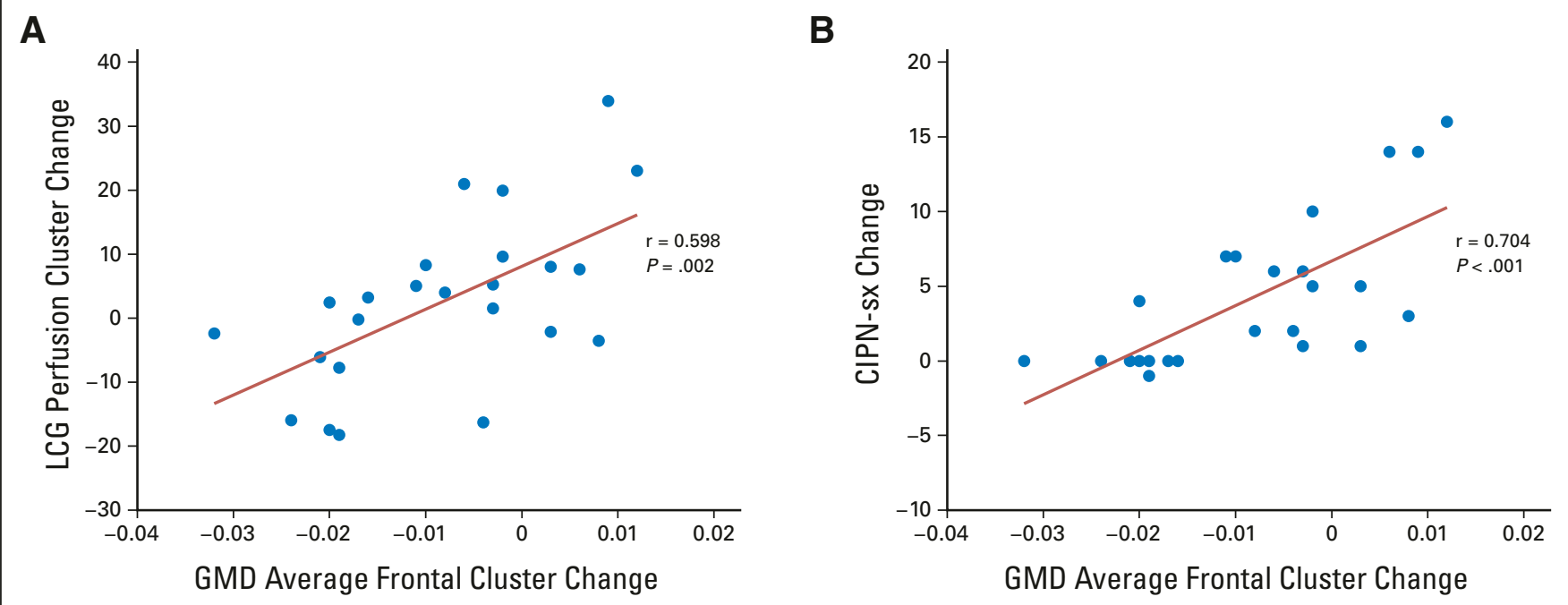

Fig 4. Gray matter density (GMD), perfusion, and chemotherapy-induced peripheral neuropathy symptom (CIPN-sx) change correlations. (A) Scatter plot of baseline (prechemotherapy treatment) to 1-month post-treatment (1M) change in GMD frontal clusters (as seen in McDonald et al ${ }^{13} ; x$-axis) and the CIPN-sx-associated perfusion change cluster in the left cingulate gyrus (LCG). These variables showed a Pearson correlation ( $r=0.598, P=.002)$, as labeled on the fit line. (B) Scatter plot of baseline to $1 \mathrm{M}$ change in GMD frontal clusters ( $x$-axis) and CIPN-sx change. These variables showed a Pearson correlation $(r=0.704, P<.001)$, as labeled on the fit line.

group, there were no significant between-group differences for both baseline and $1 \mathrm{M}$ measures of depression and anxiety. Interscan intervals (approximately 5.5 months for baseline to $1 \mathrm{M}$ and 1 year for $1 \mathrm{M}$ to $1 \mathrm{Y}$ ) did not differ significantly between groups. Finally, comparing patients who received adjuvant chemotherapy and those who received neoadjuvant chemotherapy, the eight patients who received neoadjuvant chemotherapy did not demonstrate any significant demographic differences.

\section{Treatment Group CIPN-sx Analysis}

At baseline, CIPN-sx for the chemotherapy and no chemotherapy groups were not significantly different (Table 2). At $1 \mathrm{M}$ and $1 Y$, patients in the chemotherapy group reported significantly more CIPN-sx than patients in the no chemotherapy group $(1 \mathrm{M}, P=.001$; $1 \mathrm{Y}, P=.027)$. Repeated measures analysis of all three time points for the chemotherapy group showed that these patients experienced significantly increased CIPN-sx over time $(P<.001)$. In contrast, CIPN-sx did not increase significantly over time in patients who did not receive chemotherapy $(P=.301)$. Finally, analysis of group differences in change in CIPN-sx from baseline to $1 \mathrm{M}$ showed that CIPN-sx increased significantly in chemotherapy patients compared with patients not treated with chemotherapy $(P=.001)$.

\section{CIPN-sx-Associated Perfusion in Chemotherapy- Treated Patients}

Time point analysis at $1 \mathrm{M}$ demonstrated that more CIPN-sx were associated with higher perfusion in several frontal region clusters, including bilateral superior frontal and cingulate gyri and left middle and medial frontal gyri $\left(P_{\text {unc }}<.001\right.$; Fig 2 ; Appendix Table A1, online only). However, at 1Y, analysis of CIPN-sx with perfusion did not demonstrate any significant associations.

Longitudinal analysis indicated that more CIPN-sx at $1 \mathrm{M}$, covaried for baseline, were also associated with increased perfusion from baseline to $1 \mathrm{M}$. This increase was observed in several clusters including two regions identified in the $1 \mathrm{M}$ analysis, the left cingulate gyrus and left superior frontal gyrus (Fig 3, Table A1).

\section{Gray Matter Density Correlation in Chemotherapy Patients}

Baseline to $1 \mathrm{M}$ gray matter density change was positively associated with both baseline to $1 \mathrm{M}$ CIPN-sx change and perfusion cluster change (Fig 4), indicating that individuals with more baseline to $1 \mathrm{M}$ gray matter density decrease did not tend to show increased perfusion
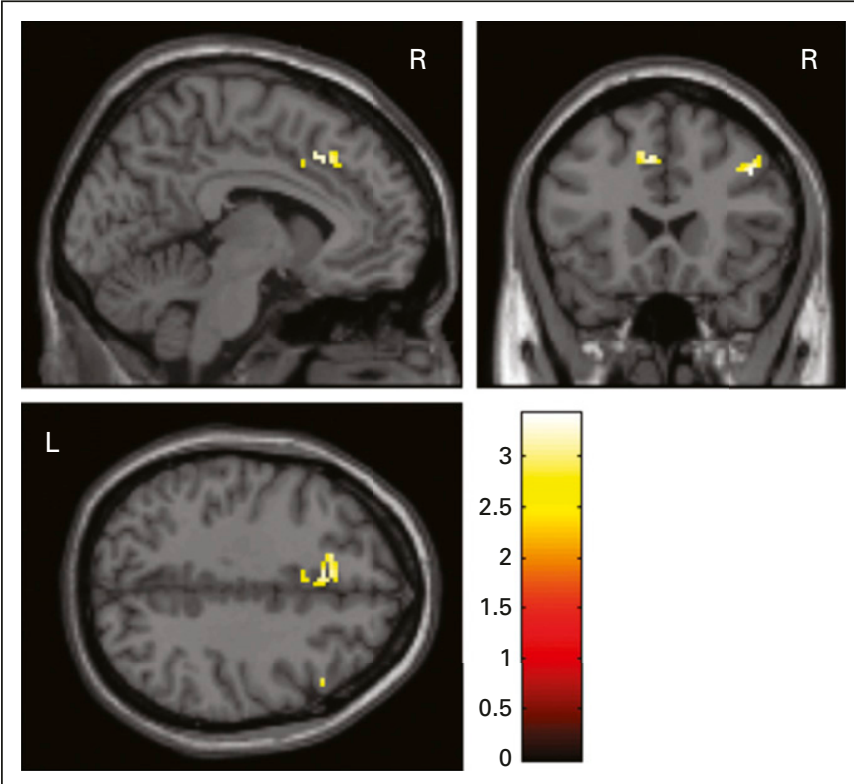

Fig 5. Cerebral perfusion decrease associated with gray matter density decrease in chemotherapy-induced peripheral neuropathy symptom-related regions of interest. A mask of regions where chemotherapy-induced peripheral neuropathy symptoms correlated with perfusion at 1 month after chemotherapy completion superimposed on the analysis presented in Nudelman et $\mathrm{al}^{14}$ of gray matter density frontal cluster change (baseline to 1 month after treatment) with perfusion change identifies clusters of overlap $\left(P_{\text {crit }}<.01, \mathrm{k}=25\right)$ in the cingulate gyrus and superior frontal gyrus; colored regions indicate increasing statistical significance, as shown on the color scale. 
and report increased CIPN-sx. Examination of the intersection of gray matter density-associated perfusion decrease and the mask of regions of perfusion associated with CIPN-sx at $1 \mathrm{M}$ showed overlap in the right superior frontal gyrus and left cingulate gyrus (Fig 5, Table A1).

\section{DISCUSSION}

To our knowledge, this is the first study to identify an association between cerebral resting state perfusion and CIPN-sx. Compared with patients who did not receive chemotherapy, patients with breast cancer treated with standard-dose chemotherapy exhibited significant post-treatment CIPN-sx. In the chemotherapy group, a significant association was observed between post-treatment CIPN-sx and perfusion in brain regions previously associated with pain processing in other populations. Specifically, the anterior cingulate region is known to be involved in pain processing, and this effect has been demonstrated in other pain populations and animal models. ${ }^{7,8,21-26}$ Preliminary evidence in the literature shows that therapies targeting this region may alleviate pain, suggesting a potential future intervention. ${ }^{27-30}$

Although a significant association between cerebral perfusion and CIPN-sx was identified at $1 \mathrm{M}$, a similar association was not observed at $1 \mathrm{Y}$, which may be partly a result of a trend toward decreased CIPN-sx at this time. Alternatively, the changes in cerebral perfusion at $1 \mathrm{M}$ may reflect an acute pain processing mechanism, perhaps distinctly different from long-term chronic pain processing mechanisms.

Given the previously reported results of frontal gray matter density decrease and associated perfusion decrease observed in this cohort, ${ }^{14}$ the impact of gray matter density change on CIPN-associated perfusion was also investigated. The association between gray matter density change, CIPN-sx, and perfusion change indicates that individuals showing gray matter density decrease may report less severe CIPN-sx while showing less CIPN-sx-related perfusion change. In this study, we also found that regions where perfusion correlated with CIPN-sx 1 month after chemotherapy completion overlapped with the previously reported ${ }^{14}$ regions where decreased gray matter density was associated with decreased perfusion. Specifically, this overlap was apparent in the left cingulate gyrus and right superior frontal gyrus, where more CIPN-sx were associated with higher perfusion and where perfusion and gray matter density were positively correlated. This suggests that decreases in gray matter density may potentially interfere with CIPN-associated perfusion increase and reduce patient symptom report. This is particularly important because symptoms such as pain, numbness, and tingling are just one component of CIPN; deficits in motor reflexes, loss of dexterity, and issues with balance are also common. If chemotherapy-treated patients with gray matter density decrease do experience (and report) fewer symptoms compared with their non-gray matter density-affected counterparts, it may mask identification and preclude intervention for potential threats to patient quality of life, occupational concerns, and CIPNrelated safety concerns such as falls and accidents in the home, particularly in the elderly population.

Several limitations to this study should be noted. First, as with many neuroimaging studies in breast cancer, heterogeneous cancer stage and treatment modalities were an unavoidable limitation that must be addressed in future research. Second, although the study protocol asked participants to self-report CIPN-sx using a validated tool, the protocol did not include CIPN evaluation and grading by a clinician. Although this information would have aided in clinical interpretation of the results and their implications, the FACT/GOGNtx has been shown to be sensitive to treatment differences and change over time ${ }^{18}$ and is comparable to other currently used scales. $^{31}$ The association of CIPN-sx with perfusion change in known pain processing regions reported here provides some convergent validation for the FACT/GOG-Ntx. A related limitation of this study is the absence of objective peripheral assessments of CIPN (eg, neurophysiologic testing, skin biopsy); filling this knowledge gap will be vital to a more comprehensive understanding of CIPNrelated neuroanatomic and functional changes.

In summary, these results identify an objective cerebral neuroimaging perfusion measure associated with CIPN-sx. Identifying functional brain regions important to pain processing in this population may suggest future intervention targets for CIPN. Furthermore, these results suggest that gray matter density decrease and associated perfusion decrease may interfere with CIPN-sxassociated perfusion change and patient symptom perception, which are potentially clinically relevant findings because this could result in patient under-reporting of CIPN-sx. Future research should use objective peripheral measures of CIPN along with neuroimaging to further elucidate this mechanism and explore potential CIPN diagnostic and clinical implications.

\section{AUTHORS' DISCLOSURES OF POTENTIAL CONFLICTS OF INTEREST}

Disclosures provided by the authors are available with this article at www.jco.org.

\section{AUTHOR CONTRIBUTIONS}

Conception and design: Brenna C. McDonald, Andrew J. Saykin Collection and assembly of data: Brenna C. McDonald, Yang Wang, Dori J. Smith, Darren P. O’Neill, Andrew J. Saykin

Data analysis and interpretation: Kelly N.H. Nudelman, Brenna C. McDonald, John D. West, Noah R. Zanville, Victoria L. Champion, Bryan P. Schneider, Andrew J. Saykin

Manuscript writing: All authors

Final approval of manuscript: All authors

\section{REFERENCES}

1. Han Y, Smith MT: Pathobiology of cancer chemotherapy-induced peripheral neuropathy (CIPN). Front Pharmacol 4:156, 2013

2. Wickham R: Chemotherapy-induced peripheral neuropathy: A review and implications for oncology nursing practice. Clin J Oncol Nurs 11: 361-376, 2007

3. Seretny M, Currie GL, Sena ES, et al: Incidence, prevalence, and predictors of chemotherapyinduced peripheral neuropathy: A systematic review and meta-analysis. Pain 155:2461-2470, 2014

4. Argyriou AA, Bruna J, Marmiroli $P$, et al: Chemotherapy-induced peripheral neuropathy
(CIPN): An update. Crit Rev Oncol Hematol 82: 51-77, 2012

5. Apkarian AV, Sosa Y, Sonty S, et al: Chronic back pain is associated with decreased prefrontal and thalamic gray matter density. J Neurosci 24: 10410-10415, 2004

6. Burgmer M, Gaubitz M, Konrad $C$, et al: Decreased gray matter volumes in the cingulo-frontal 
cortex and the amygdala in patients with fibromyalgia. Psychosom Med 71:566-573, 2009

7. Gustin SM, Peck CC, Wilcox SL, et al: Different pain, different brain: Thalamic anatomy in neuropathic and non-neuropathic chronic pain syndromes. J Neurosci 31:5956-5964, 2011

8. Lin CS: Brain signature of chronic orofacial pain: A systematic review and meta-analysis on neuroimaging research of trigeminal neuropathic pain and temporomandibular joint disorders. PLoS One 9: e94300, 2014

9. Mao CP, Zhang QL, Bao FX, et al: Decreased activation of cingulo-frontal-parietal cognitive/ attention network during an attention-demanding task in patients with chronic low back pain. Neuroradiology 56:903-912, 2014

10. Schmidt-Wilcke T, Kairys A, Ichesco E, et al: Changes in clinical pain in fibromyalgia patients correlate with changes in brain activation in the cingulate cortex in a response inhibition task. Pain Med 15:1346-1358, 2014

11. Boland EG, Selvarajah $D$, Hunter $M$, et al: Central pain processing in chronic chemotherapy-induced peripheral neuropathy: A functional magnetic resonance imaging study. PLoS One 9:e96474, 2014

12. Holohan $K N$, Von Ah D, McDonald BC, et al: Neuroimaging, cancer, and cognition: State of the knowledge. Semin Oncol Nurs 29:280-287, 2013

13. McDonald BC, Conroy SK, Smith DJ, et al: Frontal gray matter reduction after breast cancer chemotherapy and association with executive symptoms: A replication and extension study. Brain Behav Immun 30:S117-S125, 2013 (suppl)

14. Nudelman KN, Wang $Y, M c D o n a l d ~ B C$, et al: Altered cerebral blood flow one month after systemic chemotherapy for breast cancer: A prospective study using pulsed arterial spin labeling MRI perfusion. PLoS One 9:e96713, 2014

15. Barona A, Reynolds C, Chastain R: A demographically based index of pre-morbid intelligence for the WAIS-R. J Consult Clin Psych 52:885-887, 1984

16. Radloff LS: The CES-D Scale: A self-report depression scale for research in the general population. Appl Psychol Meas 1:385-401, 1977

17. Spielberger CD: State-Trait Anxiety Inventory. Palo Alta, CA Consulting Psychologists Press, 1983

18. Huang HQ, Brady MF, Cella D, et al: Validation and reduction of FACT/GOG-Ntx subscale for platinum/paclitaxel-induced neurologic symptoms: A Gynecologic Oncology Group study. Int J Gynecol Cancer 17:387-393, 2007

19. Wang Y, Saykin AJ, Pfeuffer J, et al: Regional reproducibility of pulsed arterial spin labeling perfusion imaging at 3T. Neuroimage 54:1188-1195, 2011

20. Maldjian JA, Laurienti PJ, Kraft RA, et al: An automated method for neuroanatomic and cytoarchitectonic atlas-based interrogation of $\mathrm{fMRI}$ data sets. Neuroimage 19:1233-1239, 2003

21. Peyron $R$, Laurent $B$, García-Larrea L: Functional imaging of brain responses to pain: A review and meta-analysis (2000). Neurophysiol Clin 30: 263-288, 2000

22. Yamashita $A$, Hamada $A$, Suhara $Y$, et al: Astrocytic activation in the anterior cingulate cortex is critical for sleep disorder under neuropathic pain. Synapse 68:235-247, 2014

23. Yi J, Zheng JY, Zhang W, et al: Decreased pain threshold and enhanced synaptic transmission in the anterior cingulate cortex of experimental hypothyroidism mice. Mol Pain 10:38, 2014

24. $\mathrm{Xu} \mathrm{H}$, Wu LJ, Wang $H$, et al: Presynaptic and postsynaptic amplifications of neuropathic pain in the anterior cingulate cortex. J Neurosci 28:7445-7453, 2008

25. Toyoda H, Zhao MG, Zhuo M: Enhanced quantal release of excitatory transmitter in anterior cingulate cortex of adult mice with chronic pain. Mol Pain 5:4, 2009

26. Zhuo M: Long-term potentiation in the anterior cingulate cortex and chronic pain. Philos Trans R Soc Lond B Biol Sci 369:20130146, 2014

27. Tzabazis A, Aparici CM, Rowbotham MC, et al: Shaped magnetic field pulses by multi-coil repetitive transcranial magnetic stimulation (rTMS) differentially modulate anterior cingulate cortex responses and pain in volunteers and fibromyalgia patients. Mol Pain 9:33, 2013

28. Li $X Y, K o H G$, Chen $T$, et al: Erasing injuryrelated cortical synaptic potentiation as a new treatment for chronic pain. J Mol Med 89:847-855, 2011

29. Hasan M, Whiteley J, Bresnahan R, et al: Somatosensory change and pain relief induced by repetitive transcranial magnetic stimulation in patients with central poststroke pain. Neuromodulation 17: 731-736, 2014

30. Pereira EA, Aziz TZ: Neuropathic pain and deep brain stimulation. Neurotherapeutics 11:496-507, 2014

31. Griffith KA, Merkies IS, Hill EE, et al: Measures of chemotherapy-induced peripheral neuropathy: A systematic review of psychometric properties. J Peripher Nerv Syst 15:314-325, 2010 


\section{AUTHORS' DISCLOSURES OF POTENTIAL CONFLICTS OF INTEREST}

Cerebral Perfusion and Gray Matter Changes Associated With Chemotherapy-Induced Peripheral Neuropathy

The following represents disclosure information provided by authors of this manuscript. All relationships are considered compensated. Relationships are self-held unless noted. I = Immediate Family Member, Inst = My Institution. Relationships may not relate to the subject matter of this manuscript. For more information about ASCO's conflict of interest policy, please refer to www.asco.org/rwc or jco.ascopubs.org/site/ifc.

Kelly N.H. Nudelman

No relationship to disclose

Brenna C. McDonald

Employment: Evolent Health (I)

Research Funding: GE

Yang Wang

Research Funding: Siemens

Dori J. Smith

No relationship to disclose

John D. West

No relationship to disclose

Darren P. O'Neill

No relationship to disclose

\section{Noah R. Zanville}

No relationship to disclose

Victoria L. Champion

No relationship to disclose

Bryan P. Schneider

No relationship to disclose

\section{Andrew J. Saykin}

Consulting or Advisory Role: Siemens Healthcare Diagnostics, Eli Lilly, Arkley BioTek

Travel, Accommodations, Expenses: Eli Lilly, Siemens Healthcare Diagnostics 


\section{Acknowledgment}

We thank the Indiana University Melvin and Bren Simon Cancer Center recruitment core and our oncologist colleagues for their invaluable assistance with patient recruitment and our magnetic resonance imaging research technologists for their assistance with scan acquisition. Finally, we are grateful to our participants for their time and effort; this research would not have been possible without their willingness to participate during a particularly challenging time in their lives.

\section{Appendix}

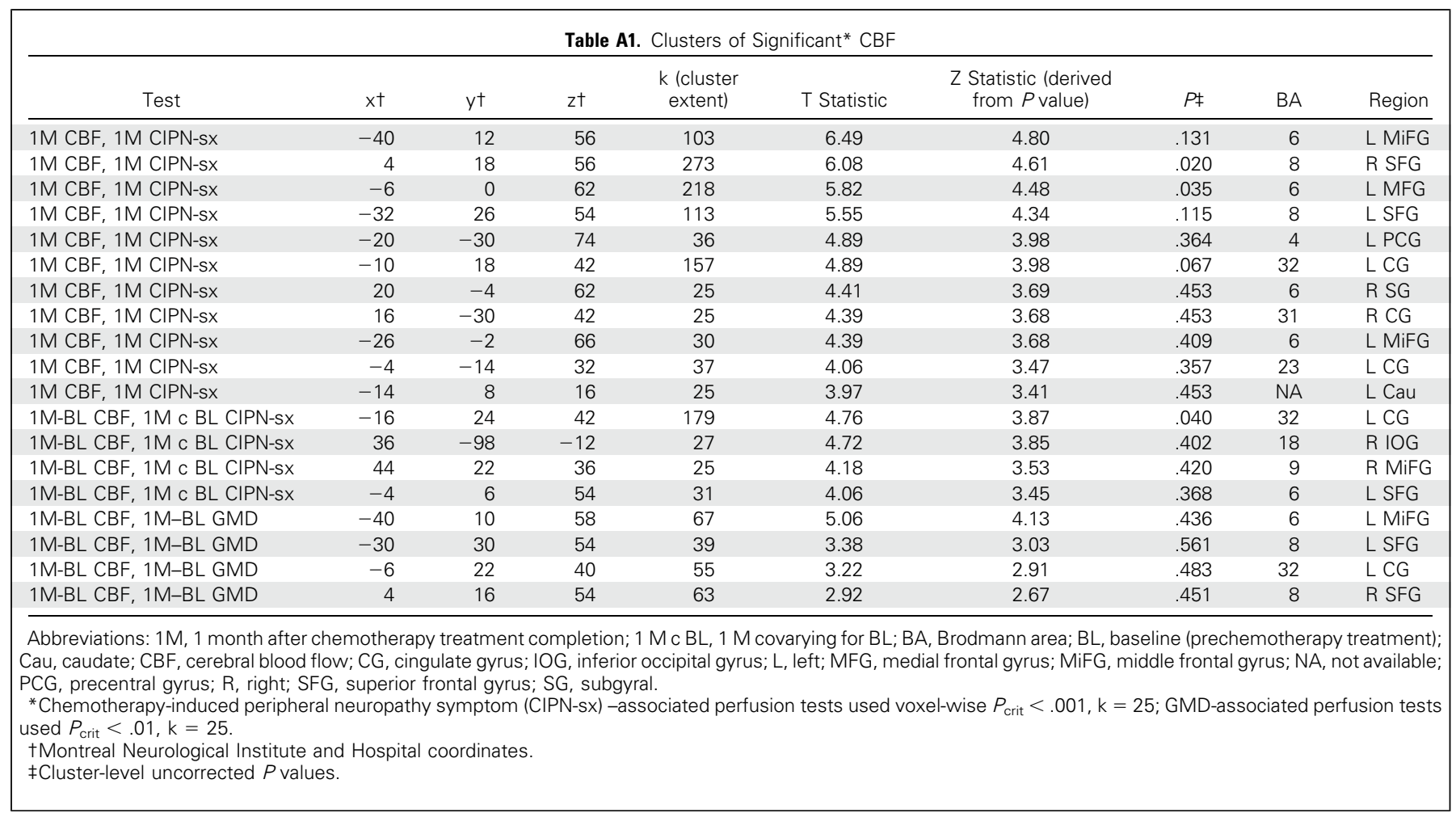

